\title{
Giant Cell Tumour of Tendon Sheath Masquerading As Trigger Finger
}

\author{
N Rahimawati, MS Orth (UKM), SA Roohi*, MS Orth (UKM), AS Naicker, M Med Rehab (UM), \\ O Zanariah*, MS Orth (UKM) \\ Universiti Kebangsaan Malaysia Medical Centre \\ *Universiti Putra Malaysia, Kuala Lumpur, Malaysia
}

\begin{abstract}
We report a case of a 59-year-old female who presented in the general orthopaedic clinic with triggering of her right middle finger. She did not respond to conventional treatment methods; subsequently she underwent surgical open release under local anaesthesia. Five months postoperatively, the patient presented with signs and symptoms of acute flexor tenosynovitis, and was thought to have a postoperative infection. Re-examination by a hand surgeon raised the possibility of a different aetiology. Based on clinical findings and response to initial treatment, giant cell tumour of the flexor tendon sheath was suspected and later confirmed following surgical biopsy. A high index of suspicion and knowledge of the variegated presentations of giant cell tumour in the hand are beneficial in these types of cases.
\end{abstract}

Key Words:

Giant Cell Tumour, Trigger Finger, Infection

\section{INTRODUCTION}

Trigger finger is a common presentation in the hand clinic and is mostly of idiopathic aetiology, but may be caused by a variety of disorders ${ }^{1}$. There are numerous reports of tumours causing trigger finger, however giant cell tumour of the tendon sheath is rarely cited as a cause in such cases ${ }^{2}$. Here we describe presentation of symptoms of infection after surgical release of trigger finger, which resulted in a delay of correct diagnosis. We are highlighting this case because a high index of suspicion is required to alert one to this condition even though it is the second most common benign soft tissue tumour of the hand ${ }^{3}$.

\section{CASE REPORT}

A 59-year-old housewife presented to the Orthopaedic Clinic in 2005 with several months history of worsening of right middle finger triggering. Clinically, there was a grade III trigger with a painless nodule over the A1 pulley. Five months later, upon failure of conservative treatment, she underwent open release under local anaesthesia. Intraoperatively, whitish, friable material was removed from the pulley. Histopathological examination revealed no evidence of inflammation or dysplasia. The triggering resolved and she was discharged from follow-up.

About 10 months after initial presentation in 2005, she presented with signs and symptoms of acute flexor tenosynovitis of one month duration at the site of the previous surgery. Laboratory tests including blood counts were within normal range. Plain radiographs of the right middle finger did not show any abnormal soft tissue or bony changes. The problem persisted despite a two-week course of oral antibiotics. She was subsequently referred to the hand clinic. Based on the clinical findings and response to initial treatment, a new working diagnosis of giant cell tumour of the flexor tendon sheath was considered. Excision biopsy under regional anaesthesia was subsequently performed. Intra-operatively, multiple yellowish nodules were noted arising from and surrounding the flexor digitorum superficialis and profundus tendons of her right middle and ring fingers (Figure 1a). The nodules extended from the midpalmar crease proximally to the A2 pulley distally. These masses adhered to the volar plate of the third metacarpophalangeal joint, the A2 pulley of the middle finger and the A1 pulley of the ring finger (Figure 1b). The digital neurovascular bundles of both fingers were spared. The affected A2 pulley had to be incised to ensure adequate clearance of the nodules (Figure 1c). No intra-osseous extension was noted. Histopathological examination confirmed the diagnosis of giant cell tumour of the tendon sheath. At the one year postoperative visit, the patient had returned to near normal range of motion and good function (Figure 2).

\section{DISCUSSION}

Giant cell tumour of the tendon sheath (GCTTS) is a rare condition with an incidence being reported at $0.5 \%{ }^{4}$. It is the 


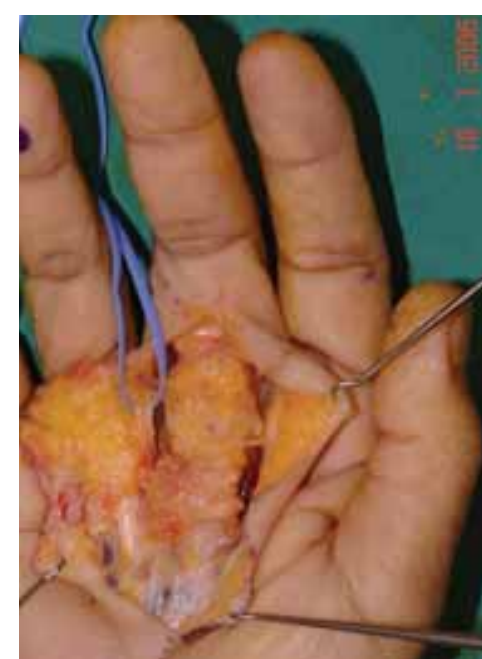

Fig. 1a: multiple yellowish nodules arising from and surrounding flexor digitorum superficialis tendon of right middle finger. Ulnar neurovascular bundle to right middle finger was isolated using a vessel loop (in blue).

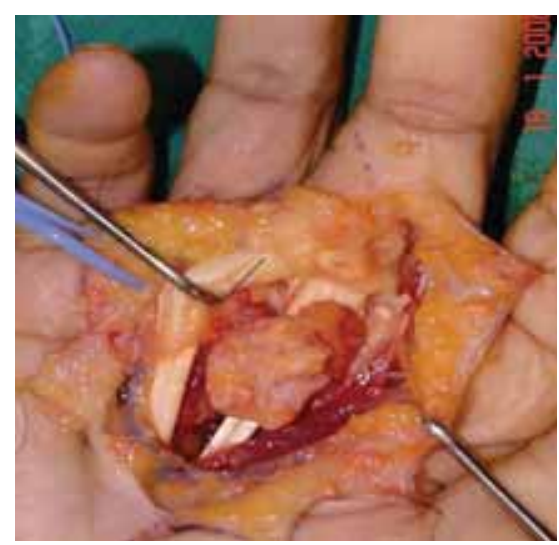

Fig. 1b: multiple yellowish nodules arising from and surrounding flexor digitorum profundus tendon of right middle finger. Flexor digitorum superficialis tendon was retracted medially using a tendon hook.
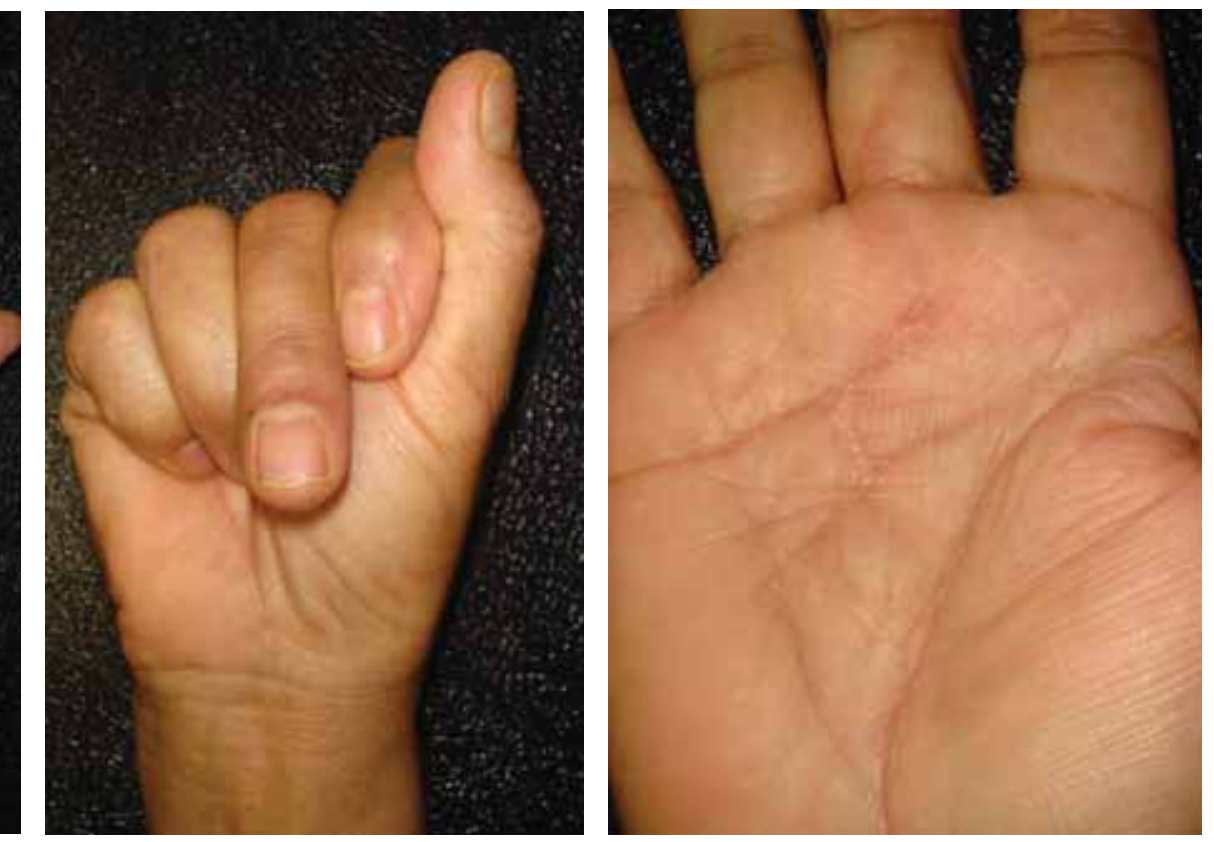

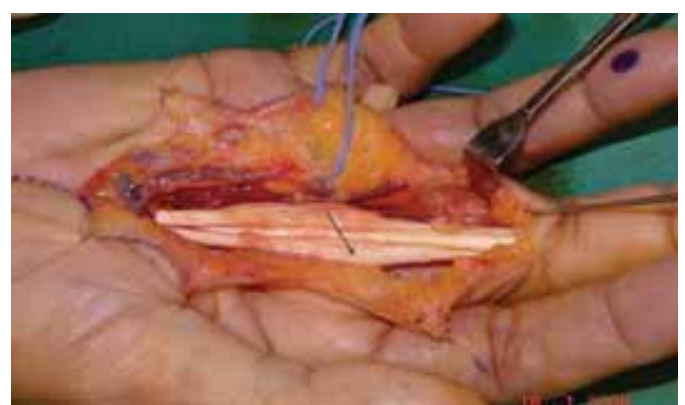

Fig. 1c: complete clearance of nodules from flexor digitorum superficialis and profundus tendons of right middle finger. Chiasma of flexor digitorum superficialis tendon can be seen clearly.

Fig. 2: Good healing of the surgical scar and near complete range of motion.

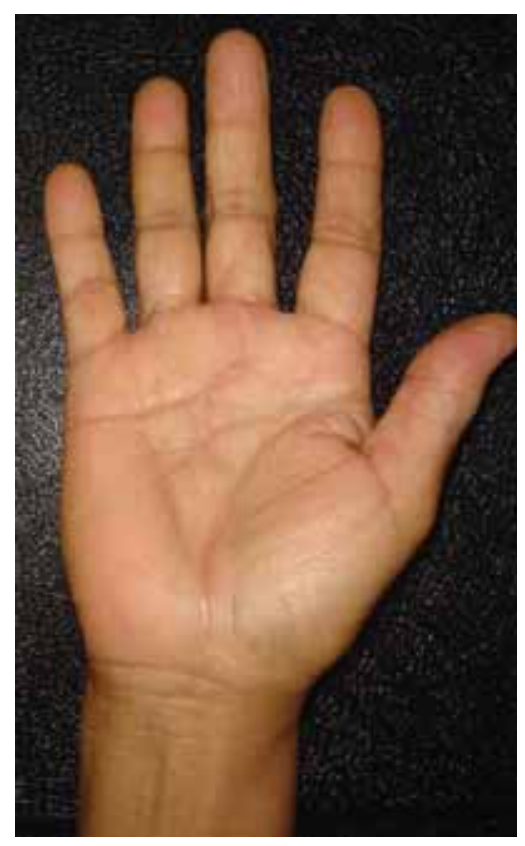


second most common soft tissue tumour of the hand and wrist, after synovial ganglion, occurring predominantly in females [4]. Localisation to the hand and wrist is noted in about $70 \%$ of cases [4], with a propensity for the radial three digits, the volar aspect and the distal interphalangeal joint.

There is usually a delay between the clinical appearance of GCTTS and the first medical consultation ${ }^{4}$, due primarily due to the absence of pain and the very slow growth of the tumour. The condition may remain static for several years. Compression of adjacent structures may occur and cause sensory disturbance (pain or numbness), snapping or triggering and restricted movement of a finger. Symptoms mimicking acute tenosynovitis may also occur. Clinically, it is often multinodular and nontender on palpation. It may be attached to deeper structures but is freely mobile underneath the skin. The tumour usually does not move with motion since it arises from the tendon sheath (and not from the flexor tendon itself), but may encircle the tendon as it did in this case.
Radiography may show erosion into adjacent bone. Sonography of volar GCTTS reveals hypoechoic tumours that are solid, with well-defined margins and irregular shape. On colour and power Doppler (with high sensitivity transducers) internal vascularity is almost always detected ${ }^{5}$.

In this patient, the whitish friable tissue may have been an indicator that this was not a normal trigger finger and perhaps should have raised suspicion for a different diagnosis. Subsequent presentation with inflammation and a distinctly palpable nodule that is mobile clinched the diagnosis. A high index of suspicion should be present at all times when a nodule is not diagnosed as a ganglion - for the next probable diagnosis should be a GCTTS. Also, as in this patient, signs of inflammation or infection may be a red herring and the surgeon must aware of other possibilities, especially if the laboratory results do not support an infection. Treatment is simple excision with care taken to preserve the neurovascular bundles and the tendons. 


\section{REFERENCES}

1. Freiberg A, Mulholland RS, Levine R. Nonoperative treatment of trigger fingers and thumbs. J Hand Surg 1989; 14(A): /553-8.

2. Stockley I and Norris S.H. Trigger finger secondary to soft tissue chondroma. J Hand Surg 1990; 15(B): 468-9.

3. Froimson AI: Benign solid tumors. Hand Clin 1987 3; 213-7.

4. Egloff DV, Ioanna D. Giant cell tumour of the tendon sheath. In: Tumors of the hand. Edited by: Egloff DV. Taylor \& Francis 2004; 27-37.

5. Teefey SA, Middleton WD and Boyer MI. Sonography of Hand and Wrist. Seminars in Ultrasound, CT and MRI 2000; 21(3): 192-204. 